hep-th/0101119

ITEP-TH-71/00

CALT 68-2306

CITUSC/00-062

HU-EP-00/54

SLAC-PUB-8749

\title{
Domain Walls, Black Holes, and Supersymmetric Quantum Mechanics
}

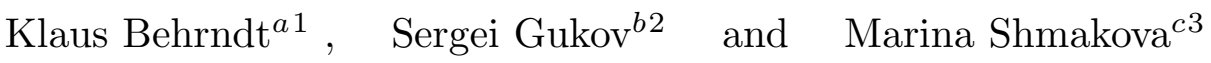 \\ ${ }^{a}$ Institut für Physik, Humboldt Universität, 10115 Berlin, Germany \\ ${ }^{b}$ Department of Physics, Caltech, Pasadena, CA 91125, USA \\ CIT-USC Center for Theoretical Physics, UCS, Los Angeles, CA 90089, USA \\ ${ }^{c}$ CIPA, 366 Cambridge Avenue Palo Alto, CA 94306, USA \\ Stanford Linear Accelerator Center, Stanford University, Stanford, CA 94309, USA
}

\begin{abstract}
Supersymmetric solutions, such as BPS domain walls or black holes, in four- and fivedimensional supergravity theories with eight supercharges can be described by effective quantum mechanics with a potential term. We show how properties of the latter theory can help us to learn about the physics of supersymmetric vacua and BPS solutions in these supergravity theories. The general approach is illustrated in a number of specific examples where scalar fields of matter multiplets take values in symmetric coset spaces.
\end{abstract}

January 2001

\footnotetext{
1 Email: behrndt@physik.hu-berlin.de

2 Email: gukov@theory.caltech.edu

3 Email: shmakova@slac.stanford.edu
} 


\section{Introduction and Summary}

Supersymmetric vacuum configurations in gauged supergravity theories in four and five dimensions recently receive a lot of attention due to their relevance to AdS/CFT correspondence [1], and, more generally, to holography in non-superconformal field theories and Domain Wall/QFT correspondence [2]. Of particular interest are BPS solutions which preserve at least four supersymmetries.

In many of such supergravity solutions, the values of scalars and other fields depend only on a single spatial coordinate. For example, in the case of a BPS black hole this is a radial coordinate, while for a domain wall this is a transverse spatial coordinate. In any case, one can integrate out the dynamics in the other directions which are isometries of the solution and obtain a $0+1$ dimensional theory - supersymmetric quantum mechanics - where the distinguished spatial coordinate plays the role of time. This relation to supersymmetric quantum mechanics was used in certain supergravity solutions, see e.g. [3]. Interpretation of the attractor flow as supersymmetric quantum mechanics was also suggested in [4].

In this work we treat both systems in a unified framework of the effective supersymmetric quantum mechanics with a certain superpotential, so that critical points of the superpotential correspond to supersymmetric (anti de Sitter) vacua in the supergravity theory. In the case of domain walls the superpotential is inherited from the original supergravity theory, and in the case of black holes it has the meaning of the central charge of a black hole. Notice, that in both cases physics requires us to extremize these quantities. Although the presence of the superpotential is crucial, a lot of interesting questions can be answered without precise knowledge of its form, simply assuming that it is generic enought.

For example, domain walls (and similarly black holes) interpolating between different minima have interpretation of the RG-flow in the holographic dual field theory on the boundary. Therefore, physically interesting questions about supersymmetric vacua and RG-flow trajectories translate into classification of ground states and gradient flows in the effective quantum mechanics. Following the seminal work of Witten [5], we use the relation between supersymmetric quantum mechanics and Morse theory to classify these supersymmetric vacuum configurations. Namely, according to Morse theory, the complex of critical points with maps induced by gradient flow trajectories is equivalent to the Hodge-de

\footnotetext{
4 Later we will explain the precise meaning of these words.
} 
Rham complex of the scalar field manifold. Notice, that the result is completely determined by the topology of the scalar field manifold, but not the form of the superpotential.

With these goals and motivation, we start in the next section with the derivation of supersymmetric quantum mechanics from five-dimensional BPS domain walls which interpolate between different $(\mathrm{AdS})$ vacua. In the dual four-dimensional theory these solutions can be interpreted as RG trajectories in the space of coupling constants. We show that these trajectories are gradient flows with a potential, so-called height function,

given by (the logarithm of) the superpotential in supergravity theory. We also explain the relation between critical points of the height function and supersymmetric vacua of the five-dimensional supergravity. In section 3 we extend these results to four-dimensional gauged supergravity, and also include interaction with hypermultiplets which has not been studied until recently. In section 4 we draw a parallel with the black hole physics and, in particular, show how quantum mechanics of the same type appears from the radial evolution. In this case, the height function is given by the central charge of the black hole. We also derive effective potentials associated with membranes wrapped over holomorphic curves in Calabi-Yau compactifications of M theory. After all these systems are reduced to supersymmetric quantum mechanics, one might hope to achieve classification of the critical points of the height function by means of Morse theory, $c f$. [5]. We review the relevant topology and explain its physical interpretation in section 5. Finally, we put all the ideas together in section 6 and demonstrate them in a family of simple examples based on $S L(3)$ symmetric coset spaces.

\section{Quantum Mechanics of Domain Walls}

BPS domain walls are kink solutions where the scalar fields interpolate between different extrema of the supergravity potential and due to the AdS/CFT correspondence, these solutions are expected to encode the RG flow of the dual field theory. Let us focus here on the 5-d case with real scalars and postpone the modifications for complex or quaternionic scalars for the next section. A Poincaré-invariant ansatz for the metric reads as follows:

$$
d s^{2}=e^{2 U}\left(-d t^{2}+d \vec{x}^{2}\right)+d y^{2}
$$

The function $U=U(y)$ is fixed by the equations of motion coming from the variation of the action:

$$
S=\int_{M}\left(\frac{R}{2}-\frac{1}{2} g_{i j} \partial \phi^{i} \partial \phi^{j}-V\right)-\int_{\partial M} K
$$


We included a surface term $K$ as the outer curvature which cancels the surface contribution from the variation of the Ricci scalar. There are no surface terms including the scalars because they asymptotically extremize the superpotential and hence are constant at the boundary. The form of the potential

$$
V=6\left(\frac{3}{4} g^{i j} \partial_{i} W \partial_{j} W-W^{2}\right)
$$

as a function of the superpotential $W$ is universal for a given dimension and follows from very general stability arguments [6]. In fact, for complex and quaternionic scalar field manifolds it is also possible to define a real superpotential $W$. In this case the supergravity potential $V$ will have the similar form [7]; see the next section.

The Poincaré invariance of the ansatz (2.1) implies that all worldvolume directions are Abelian isometries, so that we can integrate them out. For our ansatz the Ricci scalar takes the form $R=-20(\dot{U})^{2}-8 \ddot{U}$ and after a Wick rotation to an Euclidean time we find the resulting 1-dimensional action目:

$$
S \sim \int d y e^{4 U}\left[-6 \dot{U}^{2}+\frac{1}{2} g_{i j} \dot{\phi}^{i} \dot{\phi}^{j}+V\right] .
$$

In deriving this expression, the surface term in (2.2) was canceled by the total derivative term. The equations of motion of this action describe trajectories $\phi^{i}=\phi^{i}(y)$ of particles in the target space $\mathcal{M}$ with the metric $g_{i j}$. As a consequence of the 5 -d Einstein equations, these trajectories are subject to the constraint

$$
-6 \dot{U}^{2}+\frac{1}{2}\left|\dot{\phi}^{i}\right|^{2}-V=0
$$

with $\left|\dot{\phi}^{i}\right|^{2}=g_{i j} \partial_{y} \phi^{i} \partial_{y} \phi^{j}$. In order to derive the Bogomol'nyi bound we can insert the potential into (2.4) and write the action as

$$
S \sim \int d y e^{4 U}\left[-6(\dot{U} \mp W)^{2}+\frac{1}{2}\left|\dot{\phi}^{i} \pm 3 \partial^{i} W\right|^{2}\right] \mp 3 \int d y \frac{d}{d y}\left[e^{4 U} W\right]
$$

leading to the BPS equations for the function $U=U(y)$ and $\phi^{i}=\phi^{i}(y)$ :

$$
\dot{U}= \pm W \quad, \quad \dot{\phi}^{i}=\mp 3 g^{i j} \frac{\partial W}{\partial \phi^{j}}
$$

If these equations are satisfied, the bulk part of the action vanishes and only the surface term contributes. In the asymptotically $A d S_{5}$ vacuum this surface term diverges near

5 In our notation, dotted quantities always refer to $y$-derivatives. 
the AdS boundary $(U \sim y \rightarrow \infty)$ and after subtracting the divergent vacuum energy one obtains the expected result that the energy (tension) of the wall is proportional to $\Delta W_{0}=W_{+\infty}-W_{-\infty}$

Our metric ansatz (2.1) was motivated by Poincaré invariance which is not spoiled by a reparameterization of the radial coordinate. We have set $g_{y y}=1$, which is one possibility to fix this residual symmetry. On the other hand, we can also use this symmetry to solve the first BPS equation $W d y= \pm d U$, i.e. take $U$ as the new radial coordinate. In this coordinate system the metric reads

$$
d s^{2}=e^{2 U}\left(-d t^{2}+d \vec{x}^{2}\right)+\frac{d U^{2}}{W^{2}}
$$

Repeating the same steps as before we obtain the Bogomol'nyi equations for the scalars

$$
-\dot{\phi}^{i}=g^{i j} \partial_{j} \log |W|^{3}=g^{i j} \partial_{j} h
$$

which follow from the one-dimensional action

$$
S \sim \int d y\left[\left|\dot{\phi}^{i}\right|^{2}+g^{i j} \partial_{i} h \partial_{j} h\right]=\int d y\left|\dot{\phi}^{i}+g^{i j} \partial_{j} h\right|^{2}+\text { (surface term) }
$$

where $h=3 \log |W|$. As before, the field equations are subject to the constraint $\frac{1}{2}\left|\dot{\phi}^{i}\right|^{2}-$ $g^{i j} \partial_{i} h \partial_{j} h=0$ and the surface term yields the central charge. Supersymmetric vacua are given by the extrema of $h$ and the number and type of such vacua can possibly be determined by using Morse theory where $h$ is called the height function, see [5] and below.

If there are more than two smoothly connected extrema of $h$, we can build kink solutions corresponding to domain walls in the 4- or 5-dimensional supergravity. Let us summarize the different types of domain walls and discuss their implications for the RG flow and Randall-Sundrum scenario, see also [8]. As long as $W \neq 0$ at the extremum, we obtain an AdS vacuum and since the extrema of $W$ are universal (independent of the radial parameterization), we have to reach the AdS vacuum either near the boundary $(U \rightarrow+\infty)$ or near the Killing horizon $(U \rightarrow-\infty)$. Obviously, the case $U \rightarrow+\infty$ corresponds to a large supergravity length scale and therefore, due to the AdS/CFT correspondence, describes the UV region of the dual field theory. The opposite happens for $U \rightarrow-\infty$, which is related to small supergravity length scales and thus encodes the IR behavior of the dual field theory. Moreover, extrema of $W$ are fixed points of the scalar flow equations and translate into fixed points of the RG flow, which can be either UV or IR attractive. The 
universality of the fixed points of the RG flow, e.g. the scheme independence of scaling dimensions, translates in supergravity to the fact that the properties of the extrema of the superpotential are independent of the chosen parameterization of the scalar manifold.

In order to identify the different fixed points we do not need to solve the equations explicitly; as we will see, they are determined by the eigenvalues of the Hessian of the height function $h$. This data depends only on the local behavior of the superpotential near the fixed point. Let us go back to the BPS equations (2.7) and expand these equations around a given fixed point with $\left.\partial_{i} W\right|_{0}=0$ at $\phi^{i}=\phi_{0}^{i}$. The superpotential becomes

$$
W=W_{0}+\frac{1}{2}\left(\partial_{i} \partial_{i} W\right)_{0} \delta \phi^{i} \delta \phi^{j} \pm \ldots
$$

with $\delta \phi^{i}=\phi^{i}-\phi_{0}^{i}$, and the cosmological constant (inverse AdS radius) is given by $\Lambda=$ $-W_{0}^{2}=-1 / R_{A d S}^{2}$. Hence, the scalar flow equations can be approximated by 6 :

$$
\delta \dot{\phi}^{i}=-\left(g^{i j} \partial_{j} \partial_{k} W\right)_{0} \delta \phi^{k}
$$

Next, we can diagonalize the constant matrix $\left(g^{i j} \partial_{j} \partial_{k} W\right)_{0}$ and find

$$
\Omega_{k}^{i}=\left(g^{i j} \partial_{j} \partial_{k} W\right)_{0}=W_{0} \frac{1}{3} \Delta^{(i)} \delta_{k}^{i}
$$

where we absorbed the inverse length dimension into $W_{0}$. The dimensionless eigenvalues $\Delta^{(i)}$ coincide with the eigenvalues of $\partial^{i} \partial_{j} h$. According to the AdS/CFT correspondence [1], these eigenvalues are the scaling dimensions of the corresponding perturbations in the dual field theory. Namely, in a linearized version, the equations of motion for the scalars become $\partial^{2} \phi^{i}-M^{i}{ }_{j} \phi^{j}=0$ and the mass matrix reads $M^{i}{ }_{j}=\left.\partial^{i} \partial_{j} V\right|_{0}=W_{0}^{2} \Delta^{(i)}\left(\Delta^{(i)}-4\right) \delta^{i}{ }_{j}$ or, measured in the units of $W_{0}$, the mass formula becomes

$$
\left(m^{(i)}\right)^{2}=\Delta^{(i)}\left(\Delta^{(i)}-4\right)
$$

Consequently, near the AdS vacuum we find a solution to (2.11)

$$
U=\left(y-y_{0}\right) W_{0} \quad, \quad \delta \phi^{i}=e^{-\frac{1}{3} \Delta^{(i)} W_{0}\left(y-y_{0}\right)}=e^{-\frac{1}{3} \Delta^{(i)} U} .
$$

This approximate solution is, of course, valid only if $\delta \phi^{i}=\phi^{i}-\phi_{0}^{i} \rightarrow 0$ in the AdS vacuum where $U \rightarrow \pm \infty$ and therefore all eigenvalues $\Delta^{(i)}$ have the same sign: $\Delta^{(i)}>0$ for UV

6 For definiteness we took the upper sign convention. 
fixed points $(U \rightarrow+\infty)$, or $\Delta^{(i)}<0$ for IR fixed points $(U \rightarrow-\infty)$. Equivalently, UV fixed points are minima of the height function $h$ whereas IR fixed points are maxima. For this conclusion we assumed that the scalar metric has Euclidean signature and $W_{0}>0$. It is important to notice that in the definition of the scaling dimensions the matrix $\Omega^{i}{ }_{j}$ has one upper index and one lower index. It is straightforward to consider also the possibilities $W_{0}<0$ and/or timelike components of the scalar field metric. Note, the sign ambiguity in the BPS equations (2.7) interchanges both sides of the wall, i.e. it is related to the parity transformation $y \leftrightarrow-y$, which also flips the fermionic projector onto the opposite chirality.

The eigenvalues of $\Omega^{i}{ }_{k}$ of different signs mean that the extremum is IR-attractive for some scalars and UV-attractive for the other and, therefore, is not stable (a saddle point of $h$ ). Moreover, using these saddle points to connect two maxima/minima of $h$ would violate the proposed $c$-theorem for domain walls [9, 10,11,12]. Namely, multiplying eq. (2.9) by $g_{i k} \dot{\phi}^{k}$ one obtains

$$
-\dot{h}=-\dot{\phi}^{i} \partial_{i} h=g_{i j} \dot{\phi}^{i} \dot{\phi}^{j} \geq 0
$$

Therefore, along the flow, the height function $h$ has to behave strictly monotonic and at the extrema it corresponds to the central charge of the dual conformal field theory [13]: $c_{C F T} \sim R_{A D S}^{3}=1 /\left|W_{0}\right|^{3}=e^{-h_{0}}$. Recall that in our sign convention larger values of the radial parameter $U$ correspond to the UV region and are minima of the height function $h$. If we start with the UV point $(U=+\infty)$ and go towards lower values of $U$, the $c$-theorem states that $h$ has to increase, either towards an IR fixed point (maximum) or towards a positive pole in $h\left(W^{2} \rightarrow \infty\right)$, which is singular in supergravity and corresponds to $c_{C F T}=0$. On the other hand, if we start from an IR fixed point $(U=-\infty)$ and go towards larger values of $U$, due to the $c$-theorem $h$ has to decrease, either towards a minimum (UV fixed point) or towards a negative pole $\left(W^{2} \rightarrow 0\right)$, which is not singular in supergravity and corresponds to flat spacetime, $c_{C F T} \rightarrow \infty$. An example is the asymptotically flat 3-brane, where the height function parameterizes the radius of the sphere, which diverges asymptotically (indicating decompactification) and runs towards a finite value near the horizon which is IR attractive in our language.

In summary, there are the following distinct types of supergravity flows, which are classified by the type of the extremum of the height function or superpotential. Depending on the eigenvalues of the Hessian of $h$, the extrema can be IR attractive (negative eigenvalues), UV attractive (positive eigenvalues) or flat space (singular eigenvalues). Generalizing 
the above discussion and allowing also possible sign changes in $W$, the following kink solutions are possible (in analogy to the situation in four dimensions [8]):

(i) flat $\leftrightarrow \mathrm{IR}$

(ii) IR $\leftrightarrow \mathrm{IR}$

(iii) $\mathrm{IR} \leftrightarrow \mathrm{UV}$

(iv) $\mathrm{UV} \leftrightarrow \mathrm{UV} \quad$ (singular wall)

$(v) \mathrm{UV} \leftrightarrow$ singularity $\quad\left(W^{2}=\infty\right)$

Note, there is no kink solution between a UV fixed point and flat space, because the $c$ theorem requires a monotonic $h$-function and the UV point corresponds to a minimum of $h$, whereas the flat space case is a negative pole. Moreover, if there are two fixed points of the same type on each side of the wall, $W$ necessarily has to change sign implying that the wall is either singular (pole in $W$ ) or one has to pass a zero of $W$. In addition, between equal fixed points no flow is possible (that would violate the $c$-theorem) and therefore this case describes a static configuration, where the scalars do not flow. This is also what we would expect in field theory, where the RG-flows go always between different fixed points. Recall, although a zero of $W$ means a singularity in $h$, the domain wall solution can nevertheless be smooth. In models with mass deformations $\left(\Delta^{(i)}=2\right)$, type $(\mathrm{v})$ walls appear generically for models which can be embedded into maximal supersymmetric models, whereas models allowing type (iv) walls typically can not be embedded into maximal supersymmetric models, an explicit example is discussed in [14].

In the Randall-Sundrum scenario one is interested in the case (ii), because in this case the warp factor of the metric vanishes exponentially on each side of the wall.

\section{Potentials from Gauged Supergravity}

Extrema of the supergravity potential $V$ are vacua of the theory, but not all extrema correspond to stable vacua. Instead, one can show [6] that stable vacua are extrema of a superpotential $W$ which defines the supergravity potential in $d$ dimensions to be

$$
V=\frac{(d-2)(d-1)}{2}\left(\frac{d-2}{d-1} g^{i j} \partial_{i} W \partial_{j} W-W^{2}\right) \text {. }
$$

Once again, the corresponding scalar flow equations look like $\dot{\phi}^{i}=-g^{i j} \partial_{j} h$ with the height function

$$
h=(d-2) \log |W|
$$

7 Maximal supersymmetric models typically have only one UV extremum. 
We will now derive the real superpotential $W$ for different models. Depending on the number of unbroken supercharges, only special superpotentials can appear in supersymmetric models. Our primary interest are supergravity duals of field theories with 4 unbroken supercharges. Therefore the scalars on the supergravity side are part of vector, tensor or hyper multiplets that parameterize the product space:

$$
\mathcal{M}=\mathcal{M}_{V / T} \times \mathcal{M}_{H}
$$

Known potentials are related to: (i) gauging isometries of $\mathcal{M}$ or (ii) gauging the global $\mathrm{R}$ symmetry. In the first case, the scalars and fermions become charged whereas in the second case the scalars remain neutral. As a consequence of the gauging, the supersymmetry variations are altered and flat space is, in general, not a consistent vacuum. If the potential has an extremum, it is replaced by an AdS vacuum, which was not welcomed in the early days of supergravity, but fits very well in the AdS/CFT correspondence and the holographic RG flow picture. Let us discuss the different cases in more detail.

\subsection{Gauged Supergravity in 5 Dimensions}

Supergravity in five dimensions needs at least 8 supercharges, and scalar fields can be part of vector-, tensor- or hypermultiplets. Each (abelian) vector multiplet contains a $U(1)$ vector $A_{\mu}^{i}$, a gaugino $\lambda^{i}$ and a real scalar $\phi^{i}\left(i=1, \ldots, n_{V}\right)$. On the other hand, a hypermultiplet includes two hyperinos $\zeta^{u}$ and four real scalars $q^{u}\left(u=1, \ldots, 4 n_{H}\right)$. Finally, the gravity multiplet has besides the graviton, the gravitino $\psi_{m}^{A}$ and the graviphoton $A_{\mu}^{0}$.

On the vector multiplet side, supersymmetry is a powerful tool in determining allowed corrections. In fact, all couplings entering the Langrangian are fixed in terms of the cubic form [15]

$$
F=\frac{1}{6} C_{I J K} X^{I} X^{J} X^{K}
$$

In a Calabi-Yau compactification of M-theory, the fields $X^{I}\left(I=0, \ldots, n_{V}\right)$ are related to the Kähler class moduli $M^{I}$ by a rescalling $X^{I}=\frac{M^{I}}{\mathcal{V}^{1 / 3}}$ with the Calabi-Yau volume $\mathcal{V}=\frac{1}{6} C_{I J K} M^{I} M^{J} M^{K}$ and the constants $C_{I J K}$ are the topological intersection numbers [16]. The scalar fields $\phi^{a}\left(X^{I}\right)$ parameterize the space $\mathcal{M}_{V}$ defined by $F=1$ and the gaugeand scalar-couplings are given by

$$
G_{I J}=-\frac{1}{2}\left(\partial_{I} \partial_{J} F\right)_{F=1}, \quad g_{i j}=\left(\partial_{i} X^{I} \partial_{j} X^{J} G_{I J}\right)_{F=1}
$$


Much less is known on the hypermultiplet side. The four real scalars of each hypermultiplet are combined to a quaternion and parameterize a quaternionic mannifold $\mathcal{M}_{H}$. From the geometrical point of view, the hypermultiplet sector in four and five dimensional supergravity is the same, see [17] for further details. In any compactification from string or M-theory there is at least one hypermultiplet - the so-called universal hypermultiplet that contains Calabi-Yau volume $\mathcal{V}$. This name is a little bit misleading since when $n_{H}>1$ there is no unique way to single out one direction on a general quaternionic manifold [18]. As long as we have only this single hypermultiplet, its geometry is given by the coset space $\frac{S U(2,1)}{U(2)}$. But after including further hypermultiplets, quantum corrections will deform the space in a way which is rarely known.

Now let us turn to potentials resulting from gauged isometries. Both manifolds, $\mathcal{M}_{V}$ and $\mathcal{M}_{H}$, have a number of isometries [19], which can be gauged [20,21]. However the flow equations of the scalars are not sensible to gauged isometries of $\mathcal{M}_{V}$; it yields only an additional "D-flatness" constraint [22,21]. More interesting is the gauging of isometries of $\mathcal{M}_{H}$; see [23,7,24 for explicit examples. This is a quaternionic space, which implies the existence of three complex structures and an associated triplet of Kähler forms $K^{x}$. The holonomy group is $S U(2) \times S p\left(n_{H}\right)$ and the Kähler forms have to be covariantly constant with respect to the $S U(2)$ connection. The isometries are generated by a set of Killing vectors $k_{I}^{u}$

$$
q^{u} \rightarrow q^{u}+k_{I}^{u} \epsilon^{I}
$$

and the required gauge covariant derivatives become $d q^{u} \rightarrow d q^{u}+k_{I}^{u} A^{I}$. In order to keep supersymmetry, this gauging has to preserve the quaternionic structure, which means that the Killing vector has to be tri-holomorphic (in analogy with the holomorphicity in $N=1$ supergravity). This is the case if we can express them in terms of a triplet of Killing prepotentials $P_{I}^{x}$ (with the $S U(2)$ index $x=1,2,3$ )

$$
K_{u v}^{x} k_{I}^{v}=-\nabla_{u} P_{I}^{x} \equiv-\partial_{u} P_{I}^{x}-\epsilon^{x y z} \omega_{u}^{y} P_{I}^{z}
$$

Here $\omega_{u}^{y}$ are the $S U(2)$ connections related to the Kähler forms by $K_{u v}^{x}=-\nabla_{[u} \omega_{v]}^{x}$. They can be also expressed in terms of the complex structures and the quaternionic metric as: $K_{u v}^{x}=\left(J^{x}\right)_{u}^{r} h_{r v}$, and using $\sum_{x}\left(J^{x}\right)_{u}^{r}\left(J^{x}\right)_{r}^{v}=-3 \delta_{u}^{v}$ we can write the Killing vectors as $k_{I}^{u}=-\sum_{x} h^{u v}\left(J^{x}\right)_{v}^{r} \nabla_{r} P_{I}^{x}$. Next, introducing an $S U(2)$-valued superpotential

$$
W_{A}^{B} \equiv W^{x}\left(i \sigma_{x}\right)_{A}^{B} \quad \text { with : } \quad W^{x}=X^{I} P_{I}^{x}=X^{I}(\phi) P_{I}^{x}(q)
$$

8 See also below for the similar situation in 4 dimensions. 
( $\sigma_{x}$ are the Pauli matrixes) the fermionic supersymmetry variations [21] become

$$
\begin{aligned}
\delta \psi_{m}^{A} & =D_{m} \epsilon^{A}-\frac{i}{3} W_{B}^{A} \Gamma_{m} \epsilon^{B}, \\
\delta \lambda^{A i} & =-\frac{i}{2}\left[\Gamma^{m} \partial_{m} \phi^{i} \epsilon^{A}-2 i g^{i j} \partial_{j} W_{B}^{A} \epsilon^{B}\right], \\
\delta \zeta^{\alpha} & \left.=-\frac{i}{\sqrt{2}} V_{u}^{A \alpha}\left[\Gamma^{m} \partial_{m} q^{u}-2 h^{u v}\left(J^{x}\right)_{v}^{r} \nabla_{r} W^{x}\right)\right] \epsilon_{A}
\end{aligned}
$$

where $A, B$ are $S U(2)$ indices and $\partial_{i} \equiv \frac{\partial}{\partial \phi^{i}}$. We dropped the gauge field contributions, since they are not important for flat domain wall solutions; they would be important e.g. for a worldvolume geometry $R \times S_{3}$. For supersymmetric vacua, $W_{A}{ }^{B}$ has to become extremal $\left(\partial_{i} W^{x}=\nabla_{u} W^{x}=0\right.$, for all $\left.x=1,2,3\right)$. One can show [7], that the $S U(2)$ phase of $W$ does not contribute to the scalar flow equations and is absorbed by the $S U(2)$ connection entering the covariant derivative $D_{m}$. Moreover, combining all scalars $\left(\phi^{i}, q^{u}\right)$ that parameterize the space $\mathcal{M}=\mathcal{M}_{V} \times \mathcal{M}_{H}$ with the metric $g_{i j}=\operatorname{diag}\left(g_{i j}, h_{u v}\right)$ one recovers the BPS equation (2.7) with the real-valued superpotential

$$
W^{2}=\sum_{x} W^{x} W^{x}
$$

There is one especially simple example, where the superpotential is only $U(1)$ valued, i.e. the Killing prepotential has only one, say, $P_{I}^{3}$ component. In this case the $S U(2)$ covariant derivative in (3.3) becomes a partial derivative and we have the freedom to shift the Killing prepotential by any constant $P_{I}^{3} \rightarrow P_{I}^{3}+\alpha_{I}$. In fact, one can even set the Killing prepotential to zero and keep only the constants $\alpha_{I}$, which are the analogs of the FI-terms in field theory. As a consequence, the Killing vectors vanish as well as the charges of the scalars. But still, we have a non-trivial potential giving a mass to all vector scalars. In this case the superpotential becomes

$$
W^{(3)}\left(\phi^{i}\right)=\left(\alpha_{I} X^{I}\right)_{F=1}
$$

which is manifestly real valued. Due to the constraint $F=1$ this potential yields an AdS vacuum, where generically all vector-scalars are fixed and the moduli space $\mathcal{M}_{V}$ of vacua is lifted except for a discrete set of extremal points of $W^{(3)}$. From the 5-d supergravity perspective this model has been discussed in [25], and important for the RG-flow is the property 15,26

$$
\partial_{i} \partial_{j} W^{(3)}=\frac{2}{3} g_{i j} W^{(3)}+T_{i j k} \partial^{k} W^{(3)}
$$


This relation implies that all scaling dimensions, as defined in (2.12), are $\Delta^{(i)}=+2$ and fulfill a sum rule [27]: $\sum_{i} \Delta^{(i)}=2 n$, with $n=\operatorname{dim} \mathcal{M}_{V}$. Therefore, the flow is generated by mass deformations in the field theory and the positive sign indicates that all fixed points are UV attractive; IR attractive critical points are excluded for this model [22, 28]. This model can be obtained from Calabi-Yau compactification of M-theory in the presence of non-trivial $G$-fluxes parameterized by $\alpha_{I}$ [23 and the superpotential can be written as 29,30

$$
W^{(3)}=\int_{C Y} K \wedge G_{f l u x}
$$

where $K$ is the Kähler 2-form. However this compactification yields an un-stabilized Calabi-Yau volume and, as long as we treat it as a dynamical field [31,30], this compactification does not give flat space or AdS vacua. Nevertheless this run-away problem can be avoided by more general hypermultiplet gauges, see [7]. It would be interesting to derive the general $S U(2)$-valued superpotential in the same way from M-theory. For a recent study of $W^{x}$ (with $W^{3}=0$ but $W^{1} \neq 0$ and $W^{2} \neq 0$ ) see [32].

\subsection{Gauged Supergravity in 4 Dimensions}

Supergravity in 4 dimensions needs at least 4 supercharges and allows for more general (holomorphic) superpotentials which are not related to gauged isometries. In the generic case, these models have an $A d S$ vacuum with a dual 3 -d field theory with only 2 (unbroken) supercharges; for domain wall solutions see [8].

However, if we again focus our attention on models with 8 supercharges, potentials have to be related to gauged isometries. The main difference from the situation in 5 dimensions concerns the vector multiplet side. Since vector fields in four dimensions have only two on-shell degrees of freedom, each vector multiplet has to contain two scalars (in order to complete the bosonic degrees of freedom). These two real scalars can be combined into a complex scalar $z^{i}$ and supersymmetry requires that they parameterize a special Kähler space, see [17] for a review. On the other hand, the scalars $q^{u}$ entering the hypermultiplets parameterize again a quaternionic space and the gauging of the corresponding isometries goes completely analogous to the case in 5 dimensions. This time, however, it is reasonable to use the symplectic notation of special geometry and we will use the holomorphic symplectic section $\mathbf{V}=\left(X^{I}(z), F_{I}(z)\right)$ with the symplectic product defining the Kähler potential:

$$
e^{-K / 2}=\langle\overline{\mathbf{V}}, \mathbf{V}\rangle=i\left(\bar{X}^{I} F_{I}-X^{I} \bar{F}_{I}\right)
$$


Then, we obtain a similar superpotential, $c f$. eq. (3.4):

$$
W_{A}^{B} \equiv W^{x}\left(i \sigma_{x}\right)_{A}^{B} \quad \text { with } \quad W^{x}=X^{I} P_{I}^{x}=X^{I}(z) P_{I}^{x}(q) .
$$

Notice, now $X^{I}=X^{I}(z)$ is a complex field. In $N=2$ supergravity in 4 dimensions one also introduces a symplectic vector $\mathbf{F}_{\mu \nu}=\left(F_{\mu \nu}^{I}, G_{I \mu \nu}\right)$ for the gauge fields. Both gauge fields are related to each other and we took the freedom to transform all gauge fields to $F_{\mu \nu}^{I}$ and therefore only the $X^{I}$ component of the section enters $W$. As in 5 dimensions we can express the supersymmetry variations [33,17] in terms of the superpotential (3.11)

$$
\begin{aligned}
\delta \psi_{m}^{A} & =D_{m} \epsilon^{A}-\frac{i}{3} e^{K / 2} W_{B}{ }^{A} \gamma_{m} \epsilon^{B}, \\
\delta \lambda^{A i} & =-\frac{i}{2}\left[\gamma^{m} \partial_{m} z^{i} \epsilon^{A}+2 i e^{K / 2} g^{i \bar{j}} \nabla_{\bar{j}} \bar{W}_{B}^{A} \epsilon^{B}+k_{I}^{i} \bar{X}^{I} e^{K / 2} \epsilon^{A}\right], \\
\delta \zeta^{\alpha} & \left.=-\frac{i}{\sqrt{2}} V_{u}^{A \alpha}\left[\gamma^{m} \partial_{m} q^{u}-2 e^{K / 2} h^{u v}\left(J^{x}\right)_{v}{ }^{r} \nabla_{r} W^{x}\right)\right] \epsilon_{A}
\end{aligned}
$$

with $\nabla_{j} W^{x}=P_{I}^{x}(q)\left(\frac{\partial}{\partial z^{j}}+\frac{\partial K}{\partial z^{j}}\right) X^{I}(z)$ as a Kähler covariant derivative and $\nabla_{r} W^{x}$ denotes the $S U(2)$ covariant derivative, see (3.3). We included also a possible gauging of $\mathcal{M}_{V}$ related to the Killing vector $k_{I}^{i}$ and remarkably this gauging affects only the gaugino variation $\delta \lambda^{A i}$, but not the gravitino variation $\delta \psi_{m}^{A}$.

As usual the fermionic projector is derived from the timelike gravitino variation $\delta \psi_{0}^{A}$ and using the metric ansatz (2.8) this projector becomes

$$
\gamma_{U} \epsilon^{A} \pm i \frac{W_{B}{ }^{A}}{|W|} \epsilon^{B}=0
$$

and we define a real superpotential by

$$
W^{2}=e^{K}|W|^{2}=\sum_{x} e^{K} W^{x} \bar{W}^{x}=\sum_{x} e^{K} X^{I} \bar{X}^{J} P_{I}^{x} P_{J}^{x}
$$

(the hyper scalars $q^{u}$ are $4 n_{H}$ real fields so that the Killing prepotentials are real). Note, the projector (3.13) contains both, the $S U(2)$ phase as well as the $U(1)$ phase related to the complex fields $X^{I}$ and in order to ensure the vanishing of the radial gravitino variation $\delta \psi_{U}^{A}$, both phases have to be absorbed into the $S U(2)$ (resp. $U(1)$ ) Kähler connection. This can impose further constraints. 
Using the projector it is straightforward to show that the gaugino variation yields two equations?: a $D$-flatness constraint $k_{I}^{i} \bar{X}^{I}=0$ and, after fixing the sign ambiguity in the projector, the expected flow equation for the scalars $z^{i}$ :

$$
\dot{z}^{i}=-g^{i \bar{j}} \partial_{\bar{z}^{j}} \log W^{2} .
$$

Here we use: $\partial_{\bar{i}} W^{2}=2 W \partial_{\bar{i}} W=\sum_{x} e^{K} W^{x}\left(\partial_{\bar{i}}+\partial_{\bar{i}} K\right) \bar{W}^{x}=\sum_{x} \frac{W^{2}}{|W|^{2}} W^{x} \nabla_{\bar{i}} \bar{W}^{x}$. Finally, following the steps done in 5 dimensions, see also [7], the hyperino variation yields the same flow equation

$$
\dot{q}^{u}=-h^{u v} \partial_{v} \log W^{2} .
$$

As before, we can again consider the special case, where the Killing prepotentials have only one component (e.g. $P_{I}^{1}=P_{I}^{2}=0, P_{I}^{3} \neq 0$ ) and can be shifted by arbitrary constants $\alpha_{I}$. The analog of (3.7) is now the complex superpotential [17]

$$
W^{(3)}=\alpha_{I} X^{I}(z)
$$

Recall that this form is related to the special symplectic basis, where all $G_{I \mu \nu}$ gauge fields have been dualized to $F_{\mu \nu}^{I}$ gauge fields. In general, the superpotential has the covariant form [34,31]

$$
W^{(3)}=\alpha_{I} X^{I}-\beta^{I} F_{I}
$$

It allows for AdS vacua which are in one-to-one correspondence with solutions to the attractor equations, which determine extrema of the supersymmetry central charge [35, 36]. One can show that all these extrema are UV attractive [28] (due to a similar relation as (3.8)) and, therefore, cannot give regular domain wall solutions. Like in 5 dimensions, this superpotential is related to a compactification in the presence of fluxes. This time, however, it is type IIB Calabi-Yau compactification and the superpotential can be written as [31,29]

$$
W^{(3)}=\int_{C Y} \Omega \wedge H_{f l u x}
$$

where $\Omega$ is the holomorphic (3,0)-form and $H_{\text {flux }}=H_{\text {flux }}^{R R}+\tau H_{\text {flux }}^{N S}$ with $\tau$ as the complexified scalar field of type IIB string theory. Supersymmetric vacua corresponding to the

9 In this matrix equation, the coefficient in front of each Pauli matrix has to vanish. In addition, in all these calculations one has to keep in mind that the $\gamma$-matrix implicitly contains a $W$ factor due to its curved index. 
minima of this superpotential have been recently studied in [32]. Moreover, the authors of [32 pointed out a relation between these supersymmetric vacua and attractor points of $\mathcal{N}=2$ supersymmetric black holes. Namely, they demonstrated the equivalence of the supersymmetry conditions $\nabla_{i} W^{x}=0$ to the attractor equations that determine the values of the scalar fields $z^{i}$ at the horizon of a black hole. We elaborate this relation in the next section from the standpoint of the effective quantum mechanics, whereas in the rest of this section we discuss 'attractor' interpretation of the other supersymmetry condition:

$$
\nabla_{u} W^{x}=X^{I}\left(\partial_{u} P_{I}^{x}+\epsilon^{x y z} \omega_{u}^{y} P_{I}^{z}\right)=0
$$

The idea is that the supersymmetry variations of the hyperino (3.5) (or (3.12)) describe supersymmetric vacua of $\mathcal{N}=2$ gauged supergravity as well as BPS instantons (in ungauged supergravity) responsible for non-perturbative corrections to the metric on the moduli space of hypermultiplets $\mathcal{M}_{H}$. In order to see the relation more precisely, consider supergravity theory obtained from Calabi-Yau compactification of $\mathrm{M}$ theory (or type IIA string theory). Then, the instantons in questions are constructed from (bound states of membranes and) Euclidean 5-branes wrapped on the Calabi-Yau manifold [37]. Put differently, this BPS configuration can be understood as a five-brane wrapped around the Calabi-Yau space with world-volume 3-form tensor field turned on.

Apart from the number of 5-branes, the instanton is characterized by the membrane charge $\alpha_{u}$ that takes values in the homology lattice $H_{3}(C Y, \mathbb{Z})$. For a given set of the charges, one can construct a spherically symmetric solution $q^{u}(r)$ which preserves half the supersymmetry (3.5) and behaves like [37,38]:

$$
q^{u}(r) \sim \frac{\alpha_{u}}{3 r^{3}}+\text { const, } \quad r \rightarrow \infty
$$

When $r$ varies from $r=\infty$ to $r=0$, the scalar fields $q^{u}$ go to the fixed values determined by the charges $\alpha_{u}$, similar to the attractor mechanism [35]. It was shown in [37], that the radial evolution of the scalar fields $q^{u}(r)$ is described by null-geodesics in the effective $0+1$ dimensional theory with target space $\mathcal{M}_{H}$. Up to boundary terms, we expect the action of this effective theory is equivalent to the action of supersymmetric quantum mechanics (2.10) with the superpotential (3.4) (or (3.11)). Thus, we claim that the flow on the moduli space of the hypermultiplets is governed by $W^{x}$.

10 For the sake of concretness, we consider five-dimensional case obtained from compactification of $\mathrm{M}$ theory. Reduction to four dimensions is straightforward. 
Since the instanton solution is expected to be smooth at $r=0$, from the equation (3.5) (with the $U(1)_{R}$ gauge field contribution included) we find that the fixed points of the flow on the moduli space of hypermultiplets are characterized by the condition (3.17):

$$
X^{I} k_{I}^{u}=0
$$

This simple condition means that the Killing vectors obey certain linear relations at the fixed points in the space $\mathcal{M}_{H}$. Note, that in the hypermultiplet version of the attractor mechanism we have fixed limit cycles rather than fixed points11. It would be interesting to better understand the physics and the geometry of these fixed cycles in $\mathcal{M}_{H}$ [39].

Intuitively, the hypermultiplet attractor equation (3.19) could be expected by analogy with $\mathcal{N}=2$ BPS black hole solutions that exhibit enhancement of supersymmetry at the horizon. For a similar reason, one might expect enhancement of supersymmetry at the center, $r=0$, of the BPS instanton constructed from a bound state of a membrane wrapped around a special Lagrangian cycle and a five-brane wrapped on the entire CalabiYau space. As we explained in the earlier sections, a supersymmetric vacuum is given by the extremum of the superpotential (3.17) which, in turn, is equivalent to (3.19).

\section{Black Hole - Domain Wall Correspondence}

Similarity between physics of black holes of ungauged supergravity and the domain wall solutions in gauged supergravity with gauging of vector multiplets [40,41] is based on the fact that both theories can be described by the same one-dimensional effective Lagrangian. In both cases the solution is fixed by the same set of attractor equations [35,36] and the superpotential $W$ of gauged supergravity corresponds to the supersymmetry central charge $Z$.

The $\mathrm{D}=4 \mathrm{~N}=2$ action of ungauged supergravity in terms of special geometry has a well known form:

$$
\mathcal{L}=\frac{R}{2}-G_{i \bar{j}} \partial^{\mu} z^{i} \partial_{\mu} \bar{z}^{\bar{j}}-h_{u v} \partial^{\mu} q^{u} \partial_{\mu} q^{v}-\operatorname{Im} \mathcal{N}_{\Lambda \Sigma} \mathcal{F}^{\Lambda} \mathcal{F}^{\Sigma}-\operatorname{Re} \mathcal{N}_{\Lambda \Sigma} \mathcal{F}^{\Lambda *} \mathcal{F}^{\Sigma}
$$

where $z$ as in Section (3.2) are the complex scalars of vector multiplets parameterizing special Kähler manifold $\mathcal{M}_{V}$ with metric $G_{i \bar{j}}=\frac{\partial^{2} K}{\partial z^{i} \partial \bar{z}^{j}}$ and Kähler potential $K$. Their

11 We would like to thank A. Strominger for pointing this out to us and drawing our attention to the hypermultiplet version of the attractor mechanism as we describe it here. 
kinetic term can be written in the form similar to the one in (2.2): $g_{i j} d \phi^{i} d \phi^{j}=G_{i \bar{j}} \partial z^{i} \partial \bar{z}^{\bar{j}}$. The vector couplings $\operatorname{Im} \mathcal{N}$ and Re $\mathcal{N}$ depend only on scalar fields $z$. Real scalars $q^{u}$ belong to hypermultiplets and parameterize the quaternionic manifold $\mathcal{M}_{H}$ with the metric $h_{u v}$. In ungauged supergravity the hypermultiplets are decoupled from the theory and we will consider only vector multiplet scalars. We will choose the following ansatz for $\mathrm{D}=4$ extreme black hole metric [42]:

$$
d s^{2}=-e^{2 U} d t^{2}+e^{-2 U}\left(\frac{d \tau^{2}}{\tau^{4}}+\frac{1}{\tau^{2}} d \Omega_{2}^{2}\right) .
$$

This assumption leads to one-dimensional effective action of the familiar form:

$$
S_{e f f} \sim \int\left(\left(\frac{d U}{d \tau}\right)^{2}+g_{i j} \frac{d \phi^{i}}{d \tau} \frac{d \phi^{j}}{d \tau}+e^{2 U} V(\phi, p, q)\right) .
$$

The potential $V$ depends on the symplectic covariant electric and magnetic charges $\left(p^{I}, q_{I}\right)$ for symplectic vector $\mathbf{F}_{\mu \nu}=\left(F_{\mu \nu}^{I}, G_{I \mu \nu}\right)$ and can be identified with the symplectic invariant form $I_{1}$ in terms of the complex central charge (the graviphoton charge) $Z=q_{I} X^{I}-p^{I} F_{I}$ with the symplectic section $\left(X^{I}, F_{I}\right)$ :

$$
V\left(\phi^{i}, p, q\right)=I_{1}=|Z(z, p, q)|^{2}+\left|\nabla_{i} Z(z, p, q)\right|^{2}
$$

where $\nabla_{i}$ is a Kähler covariant derivative. Action (4.1) can be easily transformed to the standard form of Bogomol'nyi bound:

$$
\mathcal{L}=\left(\frac{d U}{d \tau} \pm e^{U}|Z|\right)^{2}+\left|\frac{d z}{d \tau} \pm e^{U} G^{i \bar{j}} \bar{\nabla} \bar{j} \bar{Z}\right|^{2} \pm \frac{d}{d \tau}\left[e^{U} Z\right]
$$

with the equations of motion:

$$
\frac{d U}{d \tau}= \pm e^{U}|Z|, \quad \frac{d z^{i}}{d \tau}= \pm e^{U} G^{i \bar{j}} \bar{\nabla} \bar{j} \bar{Z} .
$$

Using the same construction as in Section 3.2 for (3.15) we finally derived the equation for the scalar field $\mathrm{z}$ :

$$
\frac{d z^{i}}{d U}=G^{i \bar{j}} \partial_{\bar{j}} \log Z^{2}
$$

This form is consistent with one-dimensional supersymmetric quantum mechanics. 
For the black hole solutions in $\mathrm{D}=5$ ungauged supergravity [36,42,38] the situation is similar. The action of $\mathrm{D}=5 \mathrm{~N}=2$ ungauged supergravity coupled to vector multiplets has a form:

$$
S_{5}=\int \frac{R}{2}-\frac{1}{4} G_{I J} F^{I} F^{J}-\frac{1}{2} g_{i j} \partial \phi^{i} \partial \phi^{j}-\frac{e^{-1}}{48} \epsilon^{\mu \nu \rho \sigma \lambda} C_{I J K} F_{\mu \nu}^{J} F_{\rho \sigma}^{J} A_{\lambda}^{K} .
$$

Scalar fields $\phi^{i}$ are defined through the constraint (3.1), $F=\frac{1}{6} C_{I J K} X^{I} X^{J} X^{K}=1$, and the gauge coupling $G_{I J}$ and $g_{i j}$ are given by (3.2) and depend only on $X^{I}$ and $C_{I J K}$. The ansatz for the $\mathrm{D}=5$ black hole solutions is:

$$
d s^{2}=-e^{-4 U} d t^{2}+e^{2 U}\left(d r^{2}+r^{2} d \Omega_{3}^{2}\right),
$$

and the ansatz vector fields is: $G_{I J} F_{t r}^{I}=\frac{1}{4} \partial_{r} K_{J}$ where $K_{I}=k_{I}+\frac{Q_{I}}{r^{2}}$ and $Q^{I}$ are black hole electric charges.

Using a new radial variable $\tau=\frac{1}{r^{2}}$ and equations of motion it is easy to reduce $\mathrm{D}=5$ action to the effective $\mathrm{D}=1$ dimensional action:

$$
S_{e f f} \sim \int\left(3\left(\frac{d U}{d \tau}\right)^{2}+\frac{1}{2} g_{i j} \frac{d \phi^{i}}{d \tau} \frac{d \phi^{j}}{d \tau}+e^{4 U} \frac{1}{12} V(\phi, p, q)\right)
$$

where the potential $V$ comes from the kinetic term for the vector fields

$$
V=\frac{3}{2} Q_{I} Q_{J} G^{I J}=Z^{2}+\frac{3}{2} g^{i j} \partial Z_{i} \partial Z_{j}
$$

where $Z=X^{I} Q_{I}$ is a central charge. In (4.6) we used special geometry relations (see for example [43]): $g_{i j}=G_{I J} X_{, i}^{I} X_{, j}^{J}=-3 C_{I J} X_{, i}^{I} X_{, j}^{J}$ and $g^{i j} X_{, i}^{I} X_{, j}^{J}=G^{I J}-\frac{2}{3} X^{I} X^{J}$ where $X_{, i}^{I}=\frac{\partial X^{I}}{\partial \phi^{i}}$.

The effective action (4.6) is easily changed to the familiar BPS form:

$$
\mathcal{L}=3\left(\frac{d U}{d \tau} \pm \frac{1}{6} e^{2 U} Z\right)^{2}+\frac{1}{2}\left|\frac{d \phi^{i}}{d \tau} \pm \frac{1}{2} e^{2 U} \partial^{i} Z\right|^{2} \mp \frac{1}{2} \frac{d}{d \tau}\left[e^{2 U} Z\right]
$$

where $\left|\frac{d \phi^{i}}{d \tau}\right|^{2}=g_{i j} \frac{d \phi^{i}}{d \tau} \frac{d \phi^{j}}{d \tau}$. Once again we have a system of linear differential equations of the form:

$$
\frac{\partial U}{\partial \tau} \pm \frac{1}{6} e^{2 U} Z=0, \quad g_{i j} \frac{\partial \phi^{j}}{\partial \tau} \pm \frac{1}{2} e^{2 U} \frac{\partial Z}{\partial \phi^{i}}=0 .
$$

Using the first equation to introduce the new radial variable $d U=\frac{1}{6} e^{2 U} d \tau$ we get the familiar equation for the gradient flow in a scalar field manifold $\mathcal{M}_{V}$ :

$$
\frac{\partial \phi^{i}}{\partial U}=g^{i j} \partial_{j} \log Z^{3}
$$


This consideration shows that both cases - black holes of ungauged supergravity coupled to vector multiplets and domain walls of gauged supergravity can be treated using the same approach of one-dimensional supersymmetric quantum mechanics.

The first interesting question connected to the above discussion is the form of the black hole potential (4.6) . The same potential appears as a result of M-theory compactification on Calabi-Yau threefolds in the presence of non-trivial G-fluxes [23, 30,21] and corresponds to gauging of the universal hypermultiplet of $\mathrm{D}=5$ dimensional theory. An electrically charged five dimensional black hole corresponds to a membrane in M-theory wrapped around 2-cycles of Calabi-Yau manifold in the process of compactification. We can formally regard the M-theory compactification in the presence of the membrane-source and corresponding flux.

The 11-dimensional supergravity theory is described by the action:

$$
S_{11}=\frac{1}{2} \int_{M^{11}}\left(\sqrt{-g} R-\frac{1}{2} G \wedge * G-\frac{1}{6} C \wedge G \wedge G\right)
$$

where $C$ is a 3 -form field with 4 -form field strength $G^{(4)}=d C$. In the presence of an electrically charged membrane source Bianchi identities and equations of motion for $D=11$ theory are:

$$
\begin{gathered}
d G=0 \\
d^{\star} G=d^{*} G+\frac{1}{2} G \wedge G=2 k_{11}^{2}\left({ }^{*} J\right)
\end{gathered}
$$

where ${ }^{\star} G=\frac{\partial \mathcal{L}}{\partial G},{ }^{*}$ is Hodge-dual and $J$ is a source current with the corresponding Noether "electric" charge

$$
Q=\sqrt{2} k_{11} \int_{M_{8}}\left({ }^{*} J\right)=\frac{1}{\sqrt{2} k_{11}} \int_{S_{7}}\left({ }^{\star} G\right)^{(7)}
$$

where $M_{8}$ is a volume and $S^{7}$ is a sphere around the membrane. A formal solution to the equations of motion (4.8) reads:

$$
{ }^{\star} G={ }^{*} G+\frac{1}{2} C \wedge G=\sqrt{2} k_{11} Q \frac{\epsilon_{7}}{\Omega_{7}}
$$

where $\epsilon_{7}$ is a volume form and $\Omega_{7}$ is a 7 -volume. This solution corresponds to the vector potential of the form:

$$
C \sim \frac{Q}{\widetilde{r}^{6}} \omega_{3}
$$

where $\widetilde{r}$ is a $D=11$ transverse distance to the membrane and $\omega_{3}$ is the membrane volume form. Compactification from $D=11$ dimensions down to $D=5$ leads to the configuration when the membrane is wrapped around compact dimensions. 
A natural splitting of moduli coordinates $M^{a} \Rightarrow\left(X^{I}=\frac{M^{I}}{\mathcal{V}^{1 / 3}} ; \mathcal{V}\right)$ and the condition for function (3.1) : $F(X)=1$ defines $\left(h^{1,1}-1\right)$-dimensional hypersurface on the CalabiYau cone and $\left(h^{1,1}-1\right)$ independent coordinates $\phi^{a}\left(X^{I}\right)$ (special coordinates) on this hypersurface define vector multiplet moduli space with Kähler metric $G_{I J}(3.2)$ :

$$
G_{I J}(X)=\frac{i}{2 \mathcal{V}} \int \omega_{I} \wedge * \omega_{J}=-\left.\frac{1}{2} \partial_{I} \partial_{J} \log F(X)\right|_{F=1} .
$$

A solution (4.9) of the equations of motion take the form:

$$
G=\frac{1}{\mathcal{V}} \frac{1}{r^{3}} d t \wedge d r \wedge \alpha^{I} \omega_{I}
$$

Here $r$ is a $D=5$ transverse radial coordinate and $\mathcal{V}$ is a Clabi-Yau volume, and "electric" charges $\alpha_{I}=G_{I J} \alpha^{J}$ are

$$
\alpha_{I}=\int_{C^{(4) I} \times S_{3}}\left({ }^{*} G\right)
$$

where $C^{(4) I}, \quad I=1, \ldots, h^{1,1}$ are 4 -cycles in the Calabi-Yau manifold. The nontrivial flux of this form leads to the appearance of the nonzero scalar potential. From the $G \wedge^{*} G$ term of the action it follows that

$$
\int_{M_{11}} G \wedge^{*} G=\int_{M_{11}} \frac{\sqrt{g_{s t r}}}{r^{6}} \frac{1}{\mathcal{V}^{2}} \alpha^{I} \alpha^{J} \omega_{I} \wedge * \omega_{J}=2 \int_{M_{5}} \frac{\sqrt{g_{E}}}{r^{6}} \frac{1}{\mathcal{V}^{2}} \alpha_{I} \alpha_{J} G^{I J}(X)
$$

here we use the relation $\sqrt{g_{s t r}}=\mathcal{V}^{-\frac{5}{3}} \sqrt{g_{E}}$. The effective potential for $D=5$ is:

$$
V_{5}=\frac{1}{r^{6}} \frac{1}{\mathcal{V}^{2}} \alpha_{I} \alpha_{J} G^{I J}(X) \text {. }
$$

This $\mathrm{D}=5$ potential $V_{5}$ explicitly depends on $r$ and fells with the distance to the source so that in this case there is no run-away Calabi-Yau volume as in [23, 30].

In the effective one-dimensional theory (4.5) $U$ plays a role of a dilaton and the appearence of the effective potential (4.6) can be understood as a result of gauging of an additional axionic shift symmetry.

The consideration of Section 3 shows that hypermultiplets can also play a very important role in the physics of black holes. The difference between a black hole solution and a domain wall solution is in the presence of non-trivial vector fields in the black hole case. Those fields should be added to the supersymmetry variations (3.5) (see [21]):

$$
\begin{aligned}
\delta \psi_{m}^{A} & =D_{m} \epsilon^{A}+\frac{i}{8} X_{I}\left(\Gamma_{m}^{n p}-4 \delta_{m}^{n} \Gamma^{p}\right) F_{n p}^{I} \epsilon^{A}-\frac{i}{3} W_{B}{ }^{A} \Gamma_{m} \epsilon^{B}, \\
\delta \lambda^{A i} & =-\frac{i}{2}\left[\Gamma^{m} \partial_{m} \phi^{i} \epsilon^{A}-2 i g^{i j} \partial_{j} W_{B}^{A} \epsilon^{B}\right]+\frac{3}{8} g^{i j} \partial_{j} X_{I} \Gamma^{m n} F_{m n}^{I} \epsilon^{A}, \\
\delta \zeta^{\alpha} & \left.=-\frac{i}{\sqrt{2}} V_{u}^{A \alpha}\left[\Gamma^{m} \partial_{m} q^{u}-2 h^{u v}\left(J^{x}\right)_{v}{ }^{r} \nabla_{r} W^{x}\right)\right] \epsilon_{A} .
\end{aligned}
$$


It is easy to see that in ungauged supergravity hypermultiplets are decoupled from the theory and do not affect the solution. It may not be the case when some gauging is present.

An application of Morse theory to the black hole physics can have interesting consequences. It is possible to consider a black hole solution in gauged supergravity with vector multiplet gauging [44]. In this case hypermultiplets are also decoupled and, unfortunately (as we will see later, rather, predictably), the BPS solutions contain naked singularities or blow up near the horizon. This means that in this theory it is impossible to find a black hole with a regular horizon embedded in the $A d S_{5}$. On the other hand, application of Morse theory to this case predicts existence of only one critical point (or several of the same type) and the absence of a second non-trivial vacuum (see Section 5) is justified.

The case of the black hole solution with hypermultiplet gauging is much more complicated. In this case, hypermultiplets are no longer decoupled from the theory and the hypermultiplet gauging can lead to the appearance of a second non-trivial critical point. It will be interesting to consider such solutions in the future work.

\section{Morse Theory and Vacuum Degeneracy}

In the previous sections we studied the effective dynamics of BPS solutions in $\mathcal{N}=2$ five-dimensional supergravity which preserve the $S O(3,1)$ (or $S O(4)$ ) subgroup of the Lorentz symmetry, as well as the effective dynamics of the similar solutions in four dimensions. The examples — such as supersymmetric domain walls or spherically symmetric static black holes - correspond to solutions where space-time metric is a function of a single coordinate. Effective dynamics of a BPS state with these properties turns out to be supersymmetric quantum mechanics of the form (2.10), cf. [45, 46, 47]. The nature of the BPS state is perfectly indifferent. For all such BPS states it is true that the solution represents a gradient flow of the height function $h$ between two critical points in the scalar field manifold $\mathcal{M}$. For example, in five-dimensional gauged supergravity coupled to a certain number of vector multiplets $\mathcal{M}=\mathcal{M}_{V}$ is a Riemannian manifold parameterized by scalar fields $\phi^{A}$ from the vector multiplets and $h$ is (logarithm of) the superpotential $W$. Moreover, if this theory is obtained from compactification of M theory on a Calabi-Yau three-fold, then $\mathcal{M}_{V}$ is just the Kähler structure moduli space of this Calabi-Yau manifold, and $h$ has a microscopic interpretation in terms of a $G$-flux [23, 30]. 
In any case, the study of BPS objects described above and the classification of supersymmetric vacua in $D=4$ and $D=5$ supergravity boils down to a simpler problem in supersymmetric quantum mechanics with the effective action (2.10). This connection to supersymmetric quantum mechanics allows one to address many interesting physical questions studying more elementary system. For example, Klebanov and Tseytlin used this relation to study supergravity duals of the RG-flows in $S U(N) \times S U(N+M)$ gauge theories [3]. In this section we will discuss another application of this relation.

For a compact space $\mathcal{M}$, it has been shown by Witten [5] that supersymmetric quantum mechanics with the action (2.10) is just Hodge-de Rham theory of $\mathcal{M}$. Therefore, assuming it is also the case for certain (non-compact) scalar field manifolds $\mathcal{M}$ that occur in supergravity, we can use topological methods - in particular, Morse theory - to classify possible BPS states and supersymmetric vacua they connect. As we explained above, our results are quite generic irregardless of the physical nature of a given BPS solution; they work equally well for BPS domain walls, for spherically symmetric black holes, or any other BPS object with the effective action (2.10). We only make a couple of assumptions necessary in the following. First, we require $\mathcal{M}$ to be a Riemannian manifold, although sometimes it comes with some additional structure (e.g. complex or quaternionic structure) corresponding to additional (super-)symmetry in the problem. One has to be very careful with this assumption in the cases when $\mathcal{M}$ has singularities, in particular, in Calabi-Yau compactifications of $M$ theory. Second, we assume that the space $\mathcal{M}$ is either compact or it has the right behaviour at infinity, so that the topological methods of Morse theory are reliable 12 .

Given a Riemannian manifold $\mathcal{M}$, let $h: \mathcal{M} \rightarrow \mathbf{R}$ be a "good" Morse function of $C^{\infty}$ class. This condition means that every point in $\mathcal{M}$ is either a regular point where $d h \neq 0$ or an isolated critical point $p \in \mathcal{M}$ where $d h=0$ and $h$ can be written as:

$$
h\left(\phi^{A}\right)=h(p)-\sum_{A=1}^{k}\left(\phi^{A}\right)^{2}+\sum_{A=k+1}^{n}\left(\phi^{A}\right)^{2}
$$

in some neighborhood of $p$.

The number $k$ in (5.1) is called the Morse index of a critical point $p$ and is denoted by $\mu(p)$. It turns out that $\mu(p)$ has a nice physical interpretation in supergravity. Recall,

12 For an analog of Morse theory in complex non-compact geometry that admits a holomorphic torus action see [48]. 
that the critical points of $h$ correspond to supersymmetric vacua. Moreover, according to the analysis of section 2, the eigenvalues of the Hessian of $h$ determine the type of the attractor point. In particular, critical points with zero Morse index are UV attractive. On the other hand, critical points with $\mu=n$ are IR attractive. In general, a critical point $p$ is of mixed type, $0<\mu<n$. In models with only vector multiplets, the superpotential depends generically on all scalars and such mixed critical points do not correspond to stable vacua, see discussion after (2.14).

Therefore, in order to classify possible supersymmetric vacua, one has to know how many points have a given Morse index. Morse theory provides a nice answer to this problem in terms of the topology of the scalar field manifold $\mathcal{M}$. Before we state the result let us introduce a few more notions which will be convenient in the following.

As we explained in the previous sections, a supersymmetric domain wall or a spherically symmetric black hole corresponds to a gradient flow 13 of $h$ from one critical point $p$ to another critical point $q$. Consider the "moduli space of these BPS solutions" $M(p, q)$. Namely, for a pair of critical points $p$ and $q$ we define:

$$
M(p, q)=\left\{\phi: \mathbf{R} \rightarrow \mathcal{M} \mid \frac{d \phi}{d t}=-\nabla h, \lim _{t \rightarrow-\infty} \phi(t)=p, \lim _{t \rightarrow+\infty} \phi(t)=q\right\} / \sim
$$

to be the moduli space of the gradient trajectories from $p$ to $q$ modulo the equivalence relation $\phi(t) \sim \phi(t+$ const $)$. In general, $M(p, q)$ is not a manifold, though perturbing it a bit we can always assume that it is a manifold (or a collection of points, if we have a finite number of distinct gradient trajectories).

A classical result in Morse theory asserts that the real dimension of $M(p, q)$ is given by:

$$
\operatorname{dim} M(p, q)=\mu(p)-\mu(q)-1
$$

It immediately follows that non-singular BPS domain walls (or spherically symmetric black holes) of types (ii) and (iv) interpolating between two vacua of the same kind do not exist in theories where $h$ is a global Morse function, $c f$. the discussion in the end of section 2 . Indeed, if there are two critical points of the same type (i.e. either both IR or both UV), then the virtual dimension (5.2) becomes negative. Another physical result that follows from (5.2) is that domain walls of type (iii) connecting an IR attractive point and a UV attractive point come in $(n-1)$-dimensional families.

13 It is this place where we use the Riemannian metric on $\mathcal{M}$ to define a gradient vector field $\nabla h$. 
It is curious to note that BPS solutions interpolating between mixed (IR/UV) vacua also appear in Morse theory as boundary components of $M(p, q)$. For example, codimension 1 boundary of $M(p, q)$ consists of the points corresponding to two consequent gradient flows: first from $p$ to some other critical point $s, \mu(p)>\mu(s)>\mu(q)$, and then from $s$ to $q$ :

$$
\partial M(p, q)=\cup_{\{s\}} M(p, s) \times M(s, q) .
$$

Similarly, codimension 2 boundary consists of the points corresponding to three consequent flows $p \rightarrow s \rightarrow r \rightarrow q$ with $\mu(p)>\mu(s)>\mu(r)>\mu(q)$, etc. Including all these boundary components we obtain a compact oriented manifold $\bar{M}(p, q)$.

Now we define Witten complex $C_{*}(\mathcal{M}, h)$ as a free abelian group generated by the set of critical points of $h$ :

$$
C_{k}(\mathcal{M}, h)=\oplus_{\mu(p)=k} \mathbb{Z} \cdot[p] .
$$

Furthermore, we define $\partial: C_{k}(\mathcal{M}, h) \rightarrow C_{k-1}(\mathcal{M}, h)$ via the sum over gradient trajectories (counted with signs):

$$
\partial[p]=\sum_{\mu(q)=k-1} \# M(p, q)[q] .
$$

Then, the Morse-Thom-Smale-Witten theorem says that $\partial^{2}=0$ and:

$$
H_{*}\left(C_{*}(\mathcal{M}, h)\right)=H_{*}(\mathcal{M}, \mathbb{Z}) .
$$

Therefore, the classification of critical points and gradient trajectories which represent supersymmetric vacua and BPS solutions, respectively, can be addressed in terms of the topology of $\mathcal{M}$. In particular, one finds the following lower bound on the total number of supersymmetric vacua:

$$
\sum \operatorname{rank} C_{i}(\mathcal{M}, h) \geq \sum \operatorname{rank} H_{i}(\mathcal{M}, \mathbf{R}) .
$$

This is the classical Morse inequality.

We conclude that by using Morse theory formulas (5.2) - (5.4) one can classify fivedimensional systems discussed in sections 2 and 3. In what follows we illustrate these methods in a number of interesting examples and, in particular, we count the number of supersymmetric vacua computing the Betti numbers $b_{i}=\operatorname{rank} H_{i}(\mathcal{M}, \mathbf{R})$ of the corresponding scalar field manifolds. Even though the general formulas (5.2) - (5.4) were derived for a compact manifold $\mathcal{M}$, the Morse inequality (5.4) is expected to hold in a 
wider class of examples, including certain non-compact manifolds $\mathcal{M}_{V}$ and $\mathcal{M}_{H}$ relevant for supergravity. For example, let us consider $\mathcal{N} \geq 2$ five-dimensional gauged supergravity interacting with a certain number of vector multiplets. As we will see in a moment, such theories possess at most one supersymmetric critical point on every branch of the scalar field manifold $\mathcal{M}_{V}$, as long as interaction with other matter fields (e.g. hyper multiplets) can be consistently ignored, and as long as the superpotential $W$ is generic enough to be considered as a good global Morse function.

First, consider examples with exactly $\mathcal{N}=2$ supersymmetry where scalar fields $\phi^{A}$ take values in a real homogeneous cubic hypersurface $\mathcal{M}_{V}=\{F=1\}$ defined by (3.1) in a vector space parameterized by the fields $X^{I}$ with Minkowski signature 25]. We expect that real cubics of this form have trivial topology. Even though we do not have a mathematical proof of this fact, we argue as follows. Suppose, on the contrary, that $\mathcal{M}_{V}$ is topologically non-trivial. Then, there should exist at least two critical points, one of which must have non-zero Morse index, $0<\mu<n_{V}$. However, as we explained in section 2 , the existence of such points would violate the c-theorem [11] and contradict our original assumtion that $\mathcal{M}_{V}$ is a Riemannian manifold with a positive-definite metric.

Now let's see that scalar field manifold $\mathcal{M}_{V}$ in $\mathcal{N}>2$ five-dimensional gauged supergravity is also topologically trivial. This result immediately follows from the fact 14 that in supergravity theories with more supersymmetry the space $\mathcal{M}_{V}$ can always be represented as a quotient space of a non-compact group $G$ by its maximal compact subgroup $H$ [42]. Note, that $G$ can have time-like directions which would be inconsistent if we did not divide by $H$ that makes the metric on the quotient space $\mathcal{M}_{V}=G / H$ positive-definite. Another effect of the quotient by $H$ is that the space $G / H$ is topologically trivial unless we divide further by a discrete symmetry group, e.g. U-duality group 15 . This is one way to make the topology of $\mathcal{M}$ topologically non-trivial.

The second possibility to get theories with multiple supersymmetric vacua is to take a space of scalar fields $\mathcal{M}$ with more than one branch. For instance, an important family of theories is based on the target spaces of the following simple form:

$\underline{\mathcal{M}_{V}=S O(n, 1) / S O(n)}$ : Since $S O(n)$ is the maximal compact subgroup in the noncompact group $S O(n, 1)$ the quotient space $\mathcal{M}_{V}$ is equivalent, up to homotopy, to a set

14 We thank R. Kallosh and N. Warner for explaining this to us.

15 We are grateful to E. Witten for pointing out that a quotient by a discrete group may lead to interesting non-trivial topology of $\mathcal{M}_{V}$. 
two points. The number of points comes from the number of disconnected components in the non-compact group $S O(n, 1)$ which looks like a hyperbolic space. Therefore, irregardless of the value of $n$ in this class of models we find the following Betti numbers $b_{i}=\operatorname{rank} H_{i}\left(\mathcal{M}_{V}, \mathbf{R}\right)$ :

$$
\begin{gathered}
b_{0}=2, \\
b_{i}=0, \quad i>0 .
\end{gathered}
$$

From the Morse inequality (5.4) it follows that the corresponding supergravity theories with generic $W$ possess at least two UV attractive supersymmetric vacua. By numerical computations, one can verify that there are exactly two vacua of UV type. We remark that there are no smooth domain walls because the two vacua belong to different disconnected branches.

$\mathcal{M}_{V}=S O(n-1,1) \times S O(1,1) / S O(n-1)$ : Once again, in this example we divide a non-compact group by its maximal compact subgroup, so that the resulting space is isomorphic to a set of points for the reason explained above. This time we get 4 points, one for every disconnected component of $S O(n-1,1) \times S O(1,1)$. For the Betti numbers of $\mathcal{M}_{V}$ we obtain:

$$
\begin{gathered}
b_{0}=4, \\
b_{i}=0, \quad i>0 .
\end{gathered}
$$

Similar to the previous example, there are at least four UV attractive vacua which can not be smoothly connected by BPS domain walls.

These are examples of symmetric spaces which typically appear as scalar field manifolds in $\mathcal{N}=2$ five-dimensional supergravity theories and also in models with additional (super-)symmetry structure [49], as we mentioned earlier.

It is important to stress here that it is crucial for the height function $h$ to be globally defined over the entire target space $\mathcal{M}$. For example, this assumption breaks down in a very important class of models corresponding to $\mathrm{M}$ theory compactification on Calabi-Yau three-folds. In these models $\mathcal{M}_{V}$ is just the Kähler structure moduli space of the CalabiYau manifold. Since the Kähler structure moduli spaces usually have trivial topology, one might naively conclude from (5.3) that there is only one vacuum (a UV attractive fixed point) and no non-trivial domain walls. However, in general, $\mathcal{M}_{V}$ consists of several Kähler cones separated by the walls where certain algebraic curves in the Calabi-Yau space shrink to zero size. Local anomaly arguments in heterotic M theory suggest that $G$-flux should 
jump while crossing a Kähler wall [50]. In fact, passing through a flop transition point the second Chern class changes and, therefore, the 5-brane charge induced in the boundary field theory also jumps. Since the total 5-brane charge should be conserved (and equal to zero in the compact Horava-Witten setup) some $\alpha_{I}$ also have to jump, as if the flop curves effectively carry a magnetic charge [50]. Although the superpotential (3.9) passes these curves smoothly, because the corresponding $X^{I}$ vanishes there, its (second) derivatives jump once we cross a Kähler wall, and the corresponding $h$ is not a globally defined height function.

A similar result occurs in gauged supergravity theories coupled to hyper multiplets. In this case supersymmetry implies that scalar fields from hyper multiplets parameterize a quaternionic manifold $\mathcal{M}_{H}$ of negative curvature [51]:

$$
R=-8\left(n_{H}^{2}+2 n_{H}\right)
$$

Even though this condition is not as much restrictive as the supersymmetry condition (3.8) in the case of vector multiplets, known examples of quaternionic homogeneous coset spaces that may serve as hypermultiplet target manifolds are typically non-compact and topologically trivial:

$\mathcal{M}_{H}=S O(n, 4) / S O(n) \times S O(4)$ : Similar to the example $\mathcal{M}=S O(n, 1) / S O(n)$, this quaternionic space is contractible since $16 S O(n) \times S O(4)$ is the maximal compact subgroup of $S O(n, 4)$. Therefore, $\mathcal{M}_{H}$ is homologous to a set of two points:

$$
\begin{gathered}
b_{0}=2, \\
b_{i}=0, \quad i>0 .
\end{gathered}
$$

The same result we find for $\mathcal{M}_{H}=S U(n, 2) / S U(n) \times S U(2)$. As we mentioned earlier, a simple way to obtain models where scalar fields take values in a topologically non-trivial manifold $\mathcal{M}_{H}$ is to devide by a discrete group which, for example, may be a subgroup of the isometries of $\mathcal{M}_{H}$. For example, if we have only the universal hypermultiplet, $n=1$, the coset space $\mathcal{M}_{H}=S U(1,2) / U(1) \times S U(2)$ is a quaternionic Kähler manifold, where the Kähler potential can be written as $K(S, C)=-\log \left(S+\bar{S}-2(C+\bar{C})^{2}\right)$. It has two abelian isometries corresponding to shifts of the complex scalar fields $S$ and $C, S \rightarrow S+i a$

16 Stricty speaking, $S(O(n) \times O(4))$ is the maximal compact subgroup of $S O(n, 4)$. 
and $C \rightarrow C+i b$. To get a topologically non-trivial manifold, we can consider a quotient space:

$$
\mathcal{M}_{H} / \mathbb{Z}^{2}=\frac{S U(1,2)}{U(1) \times S U(2) \times \mathbb{Z}^{2}}
$$

where the action of $\mathbb{Z}^{2}$ is equivalent to identification $S \sim S+i a$ and $C \sim C+i b$ for integer numbers $a$ and $b$. Supergravity theory coupled to a hypermultiplet based on the resulting quotient space is expected to have at least $\sum_{i} b_{i}\left(\mathcal{M}_{H} / \mathbb{Z}^{2}\right)=4$ supersymmetric vacua.

Before we conclude this section, let us remark that due to its construction, the superpotential or height function may not depend on all hyper-scalars and the chosen gauged isometry of $\mathcal{M}_{H}$ determines the scalars on which the superpotential depends. Hence, for a given superpotential, obtained by a specific gauging, a mixed critical point with $0<\mu<n_{H}$ may appear as a "good" UV or IR fixed point. Only the critical points with $\mu=0$ and $\mu=n_{H}$ are "gauge-independent" and appear in all gaugings as UV and IR fixed points. Assuming that Morse inequalities are saturated, every component of $\mathcal{M}$ has exactly one UV critical point with $\mu=0$ and at most one IR point with $\mu=n_{H}$ if $\mathcal{M}$ is a compact manifold 17 . The values of all scalar fields are fixed in these two critical points. Additional (mixed) critical points can be stable under the UV/IR scaling only if the superpotential has (bad) flat directions.

\section{Examples: $S L(3)$ Symmetric Coset Spaces}

Let us consider in more detail a specific example where the quotient space which appears in $\mathcal{N}=2$ five-dimensional supergravity is associated with Jordan algebras of the form:

$$
\mathcal{M}=\frac{\operatorname{Str}_{0}(\mathrm{~J})}{\operatorname{Aut}(\mathrm{J})}
$$

where $\operatorname{Str}_{0}(J)$ is the reduced structure group and $\operatorname{Aut}(J)$ is the automorphism group of a real unital Jordan algebra of degree 3. A simple example based on irreducible $J$ is $\mathcal{M}=S L(3, \mathbf{R}) / S O(3)$, which is a three-dimensional analog of the Lobachewsky plane, $S L(2, \mathbf{R}) / S O(2)$. Like its two-dimensional analog, the space $\mathcal{M}$ is contractible because a semisimple Lie group $S L(n, \mathbf{R})$ is isomorphic to its maximal compact subgroup $S O(n)$. After we divide by the latter we get a point, up to homotopy:

$$
b_{0}=1
$$

17 It should be stressed, however, that the authors do not know whether supergravity theories based on compact scalar field manifolds $\mathcal{M}$ exist or not. 


$$
b_{i}=0, \quad i>0
$$

So, we come to the conclusion that the STU model with generic superpotential has a single supersymmetric vacuum. In the rest of this section our goal will be to identify the physics of this vacuum.

There are two generalizations of this example over complex numbers and quaternions. In any case we divide $S L(3, F)$, where $F=\{\mathbf{R}, \mathbf{C}, \mathbf{H}\}$ is the base field in question, by its maximal compact subgroup. Although the resulting quotient space has trivial topology (6.1) for all ground fields $F$, the physics is different. Namely, we claim that three supersymmetric vacua corresponding to various choices of $F$ describe $\operatorname{AdS}_{5}$ dual of $\mathcal{N}=4$ super-Yang-Mills perturbed by mass terms which preserve different subgroups of $S O(6)$ R-symmetry.

In order to see that a scalar vev. gives rise to a mass deformation, one has to find scaling dimension $\Delta^{(i)}$ of the corresponding operator $\mathcal{O}^{(i)}$ in the boundary theory. Using the standard formula $(2.13)$ we get $\Delta^{(i)}=2$ which allows us to identify $\mathcal{O}^{(i)}$ with a mass term. In general, a mass term is specified by a bosonic symmetric $6 \times 6$ matrix and a fermionic symmetric $4 \times 4$ matrix. In our three exampes, however, these matrices have additional symmetries reminiscent of $\mathcal{N}=4$ super-Yang-Mills theory with R-symmetry twists [52]. In particular, at special values of twists, where the corresponding phases are equal to $(-1)$, extra hypermultiplets become massless 18 , and we expect to get a fourdimensional superconformal theory 19.

Below we discuss in more details the case where $F=\mathbf{C}$. In particular, we solve the flow equations (2.7) for the coset space $S L(3, \mathbf{C}) / S U(3)$. This coset manifold is parameterized by 8 non-trivial scalar fields with the intersection form [15]

$$
F=S T U-S|X|^{2}-T|Y|^{2}-U|Z|^{2}+2 \operatorname{Re}(X Y Z)
$$

18 To see this, it is convenient to think of gauge theories with R-symmetry twists as compactifications of five-dimensional gauge theories on a circle with twisted boundary conditions on $S^{1}$ [52]. The masses of Kaluza-Klein states are given by $m=n+1 / 2+q W$ where $W$ is the value of the Wilson lines of the gauge field and $q$ is the charge of a given mode. Note that for any $n \in \mathbb{Z}$ we get a whole hyper-multiplet. Moreover, for $n=0$ and -1 and $W=1 / 2$ and $q=+1$ and -1 we find two massless hypermultiplets.

19 We thank Ori Ganor for discussions on this point. 
where $X, Y$ and $Z$ are complex and $S, T$ and $U$ are real. The unique solution of the attractor equations [36]: $\partial_{I} F=e^{-2 U} H_{I}$, which solves the flow equations (2.7) (see [30]) is given

$$
\begin{aligned}
S & =\left(H_{T} H_{U}-\frac{1}{4}\left|H_{X}\right|^{2}\right) e^{-4 U}, & X & =\frac{1}{4}\left(\bar{H}_{Y} \bar{H}_{Z}-2 H_{X} H_{S}\right) e^{-4 U}, \\
T & =\left(H_{S} H_{U}-\frac{1}{4}\left|H_{Y}\right|^{2}\right) e^{-4 U}, & Y & =\frac{1}{4}\left(\bar{H}_{X} \bar{H}_{Z}-2 H_{Y} H_{T}\right) e^{-4 U}, \\
U & =\left(H_{S} H_{T}-\frac{1}{4}\left|H_{Z}\right|^{2}\right) e^{-4 U}, & Z & =\frac{1}{4}\left(\bar{H}_{X} \bar{H}_{Y}-2 H_{Z} H_{U}\right) e^{-4 U}
\end{aligned}
$$

where $H_{I}$ is a set of harmonic functions

$$
H_{I}=h_{I}+6 \alpha_{I} y
$$

which are real for the $S, T, U$ components and complex for the $X, Y, Z$ components. For the metric we take the ansatz:

$$
d s^{2}=e^{2 U}\left[-d t^{2}+d \vec{x}^{2}\right]+e^{-4 U} d y^{2}
$$

where the function $e^{-2 U}$ is obtained from the requirement $F=1$ :

$$
e^{6 U}=H_{S} H_{T} H_{U}-\frac{1}{4}\left(H_{S}\left|H_{X}\right|^{2}+H_{T}\left|H_{Y}\right|^{2}+H_{U}\left|H_{Z}\right|^{2}\right)+\frac{1}{4} \operatorname{Re}\left(H_{X} H_{Y} H_{Z}\right) .
$$

The scalar fields are defined by $F=1$ and we may consider, for example, the ratios: $\phi^{A}=\left\{\frac{T}{S}, \frac{U}{S}, \frac{X}{S}, \frac{Y}{S}, \frac{Z}{S}\right\}$. The uniqueness of the solution fits very well with our expectation from Morse theory, that this coset allows only a unique critical point. At the critical point the space time becomes $A d S_{5}$ as $y \rightarrow+\infty$ with the negative cosmological constant:

$$
\Lambda=-\left(e^{4 U} / y^{2}\right)_{y \rightarrow+\infty}
$$

In the case of a unique critical point with a negative cosmological constant, it is natural to ask what is the four-dimensional field theory dual to this $\mathrm{AdS}_{5}$ vacuum.

Notice that the scalar fields $\phi^{A}$ defined as ratios of harmonic functions stay finite in the AdS vacuum and approach their critical values. Having the explicit solution, we can also calculate the supergravity effective action. As given by (2.6), the BPS nature ensures vanishing of the bulk term and the surface part yields20.

$$
S_{e f f}=\frac{2}{3}\left(\partial_{y} e^{6 U}\right)_{y=+\infty}=\left(-2 \Lambda y^{2}+a_{1} y\right)_{y \rightarrow \infty}+a_{2} .
$$

20 Note the different coordinate system. 
As expected, this action has singular terms and a finite part. The leading singularity scales with the cosmological constant (AdS volume) while the subleading term $a_{1}$ and the finite term $a_{2}$ can be obtained by inserting the harmonic functions (6.5). The divergent part will be subtracted by the renormalization in the field theory and the finite part will give the renormalized effective action; in our approximation we see only potential or mass terms.

From the RG-flow point of view this AdS vacuum corresponds to an UV fixed point. While moving towards negative $y$ the warp factor $e^{2 U}$ decreases monotonically (according to $c$-theorem) and we approach the IR region in the fields theory. Because $e^{6 U}$ is negative at $y=-\infty$, we have to pass a zero at some finite value of $y$, which is the singular end-point of the RG flow. Like in any other case with vector multiplets only, the absence of an IR fixed point forces the solution to run into a singularity.

On the other hand, from the string theory perspective this solution corresponds to a 5-brane wrapping a holomorphic 2-cycle, namely a torus $T^{2}$. Once again, we point out the analogy with the construction of gauge theories with R-symmetry twists from $(2,0)$ theory on a torus in the limit where the size of the torus and the values of twists tend to zero $\left(\operatorname{Vol}\left(T^{2}\right) \rightarrow 0\right.$ and $\left.\alpha \rightarrow 0\right)$ while their ratio $\alpha / \operatorname{Vol}\left(T^{2}\right)$ remains finite and defines a mass scale in the resulting theory [52]. From this construction it is clear that at the special values of twists $\alpha$, where the theory becomes superconformal, it must be dual to $\operatorname{AdS}_{5} \times S_{5}$ perturbed by a dimension- 8 operator (proportional to $\operatorname{Vol}\left(T^{2}\right)$ ) and dimension-2 operators (proportional to $\alpha$ ), similar to the $\mathrm{AdS}_{5}$ vacuum of $S L(3)$ coset spaces we found.

It is reasonable to put another (5-brane) source at some place where the warp factor is still positive, say $y=0$. This extra source appears as a non-trivial right-hand side of the harmonic equations:

$$
\partial^{2} H_{I} \sim \alpha_{I} \delta(y)
$$

where $\alpha_{I}$ is the component of the 5-brane charge related to a basis of $(1,1)$-forms $\omega_{I}$. There are two possibilities: in the first option we continue in a symmetric way through the source, which implies the replacement $H_{I} \rightarrow h_{I}+6 \alpha_{I}|y|$ and is equivalent to a sign change in the flux vector $\alpha_{I}$ while passing the source at $y=0$. This case appears in the $\mathbb{Z}_{2}$ orbifold of the Horava-Witten setup compactified to 5 dimensions [23]. But we may also consider the case where the flux jumps from zero to a finite value, i.e. on the side behind the source we can set $\alpha_{I}=0$, which is equivalent to the replacement $H \rightarrow h_{I}+6 \alpha_{I} \frac{1}{2}(y+|y|)$, so that $H_{I}$ is constant for negative $y$ and the space time is flat.

By adding this source, we cut off the (singular) part of space-time and glue instead an identical piece $\left(Z_{2}\right.$ symmetric) or flat space (vanishing flux on one side). If one wants 
to discuss a RS-type scenario, one may also cut off the regular AdS part and keep on both sides the naked singularity. In the first case the source is the standard positive tension brane generating an AdS space on both sides, whereas in the second case a negative tension brane is accompanied with a naked singularity. For a more detailed discussion of sources see [53].

\section{Acknowledgments}

We are grateful Alexander Chervov, Ori Ganor, Brian Greene, Renata Kallosh, Eric Sharpe, Gary Shiu, Andrew Strominger, Nicholas Warner, and Edward Witten for helpful discussions and comments. The work of K.B. was partly done at the Theory group of Caltech and is supported by a Heisenberg grant of the DFG and by the European Commission RTN programme HPRN-CT-2000-00131. S.G. is supported in part by the Caltech Discovery Fund, grant RFBR No 98-01-00327 and Russian President's grant No 00-15-99296. 


\section{References}

[1] J.M. Maldacena, "The Large N Limit of Superconformal Field Theories and Supergravity", Adv. Theor. Math. Phys. 2 (1998) 231; S.S. Gubser, I. R. Klebanov, A. M. Polyakov, "Gauge Theory Correlators from Non-Critical String Theory", Phys. Lett. B428 (1998) 105; E. Witten, "Anti De Sitter Space And Holography", Adv. Theor. Math. Phys. 2 (1998) 253.

[2] H.J. Boonstra, K. Skenderis, P.K. Townsend, "The domain-wall/QFT correspondence", JHEP 9901 (1999) 003.

[3] I.R. Klebanov, A.A. Tseytlin, "Gravity Duals of Supersymmetric SU(N) x SU(N+M) Gauge Theories," Nucl.Phys. B578 (2000) 123.

[4] G. Moore, "Arithmetic and Attractors," hep-th/9807087.

[5] E. Witten, "Supersymmetry and Morse theory", J.Diff.Geom. 17(1982) 661.

[6] P.K. Townsend, "Positive energy and the scalar potential in higher dimensional (super) gravity", Phys. Lett. B148, 55 (1984).

[7] K. Behrndt, C. Herrmann, J. Louis and S. Thomas, "Domain walls in five dimensional supergravity with non-trivial hypermultiplets," hep-th/0008112.

[8] M. Cvetič and S. Griffies, "Domain walls in N=1 supergravity", hep-th/9209117; M. Cvetic and H. H. Soleng, "Supergravity domain walls," Phys. Rept. 282 (1997) 159, hep-th/9604090].

[9] E. Alvarez and C. Gomez, "Geometric holography, the renormalization group and the c-theorem," Nucl. Phys. B541 (1999) 441, hep-th/9807226.

[10] L. Girardello, M. Petrini, M. Porrati and A. Zaffaroni, "Novel local CFT and exact results on perturbations of $\mathrm{N}=4$ super Yang-Mills from AdS dynamics," JHEP 9812 (1998) 022 hep-th/9810126.

[11] D.Z. Freedman, S.S. Gubser, K. Pilch and N.P. Warner, "Renormalization group flow from holography, supersymmetry and a c-theorem", hep-th/9904017.

[12] K. Behrndt, "Domain walls of $\mathrm{D}=5$ supergravity and fixed points of $\mathrm{N}=1$ super Yang-Mills," Nucl. Phys. B573 (2000) 127, hep-th/9907070.

[13] M. Henningson and K. Skenderis, "Holography and the Weyl anomaly" JHEP 9807, 023 (1998), hep-th/9812032.

[14] K. Behrndt and M. Cvetic, "Supersymmetric domain wall world from D $=5$ simple gauged supergravity," Phys. Lett. B475, 253 (2000), hep-th/9909058.

[15] M. Gunaydin, G. Sierra and P. K. Townsend, "The geometry of N=2 Maxwell-Einstein supergravity and Jordan algebras", Nucl. Phys. B242, 244 (1984).

[16] A. C. Cadavid, A. Ceresole, R. D'Auria and S. Ferrara, "Eleven-dimensional supergravity compactified on Calabi-Yau threefolds," Phys. Lett. B357, 76 (1995), hepth/9506144. 
[17] L. Andrianopoli, M. Bertolini, A. Ceresole, R. D'Auria, S. Ferrara, P. Fre and T. Magri, "N $=2$ supergravity and $\mathrm{N}=2$ super Yang-Mills theory on general scalar manifolds: Symplectic covariance, gaugings and the momentum map," J. Geom. Phys. 23, 111 (1997) hep-th/9605032.

[18] P. Aspinwall, private communication.

[19] B. de Wit and A. Van Proeyen, "Isometries of special manifolds," hep-th/9505097

[20] M. Gunaydin and M. Zagermann, "The gauging of five-dimensional, N = 2 MaxwellEinstein supergravity theories coupled to tensor multiplets," Nucl. Phys. B572 (2000) 131, hep-th/9912027; "The vacua of 5d, N = 2 gauged Yang-Mills/Einstein/tensor supergravity: Abelian case," Phys. Rev. D62 (2000) 044028, hep-th/0002228.

[21] A. Ceresole and G. Dall'Agata, "General matter coupled N =2, D = 5 gauged supergravity," hep-th/0004111.

[22] R. Kallosh and A. Linde, "Supersymmetry and the brane world," JHEP 0002, 005 (2000), hep-th/0001071.

[23] A. Lukas, B. A. Ovrut, K. S. Stelle and D. Waldram, "Heterotic M-theory in five dimensions," Nucl. Phys. B552, 246 (1999), hep-th/9806051.

[24] K. Behrndt and M. Cvetic, "Gauging of $\mathrm{N}=2$ supergravity hypermultiplet and novel renormalization group flows," hep-th/0101007.

[25] M. Gunaydin, G. Sierra and P. K. Townsend, "Gauging The D = 5 Maxwell-Einstein Supergravity Theories: More On Jordan Algebras", Nucl. Phys. B253, 573 (1985).

[26] A. Chou, R. Kallosh, J. Rahmfeld, S. Rey, M. Shmakova and W. K. Wong, "Critical points and phase transitions in 5d compactifications of M-theory," Nucl. Phys. B508, 147 (1997), hep-th/9704142.

[27] A. Khavaev and N. P. Warner, "A class of $\mathrm{N}=1$ supersymmetric RG flows from five-dimensional $\mathrm{N}=8$ supergravity," hep-th/0009159.

[28] K. Behrndt and M. Cvetic, "Anti-de Sitter vacua of gauged supergravities with 8 supercharges," Phys. Rev. D61, 101901 (2000), hep-th/0001159.

[29] S. Gukov, "Solitons, superpotentials and calibrations," Nucl. Phys. B574, 169 (2000), hep-th/9911011.

[30] K. Behrndt and S. Gukov, "Domain walls and superpotentials from M theory on Calabi-Yau three-folds", Nucl. Phys. B580, 225 (2000), hep-th/0001082.

[31] T. R. Taylor and C. Vafa, "RR flux on Calabi-Yau and partial supersymmetry breaking," Phys. Lett. B474, 130 (2000), hep-th/9912152.

[32] G. Curio, A. Klemm, D. Luest, S. Theisen, "On the Vacuum Structure of Type II String Compactifications on Calabi-Yau Spaces with H-Fluxes," hep-th/0012213.

[33] B. de Wit, P. G. Lauwers and A. Van Proeyen, "Lagrangians Of $\mathrm{N}=2$ Supergravity Matter Systems," Nucl. Phys. B255, 569 (1985).

[34] J. Michelson, "Compactifications of type IIB strings to four dimensions with nontrivial classical potential," Nucl. Phys. B495, 127 (1997), hep-th/9610151. 
[35] S. Ferrara, R. Kallosh and A. Strominger, "N=2 extremal black holes," Phys. Rev. D52 (1995) 5412, hep-th/9508072

[36] S. Ferrara and R. Kallosh, "Supersymmetry and attractors," Phys. Rev. D54 (1996) 1514, hep-th/9602136

[37] M. Gutperle, M. Spalinski, "Supergravity Instantons for N=2 Hypermultiplets," hep-th/0010192.

[38] W. A. Sabra, "Black holes in $\mathrm{N}=2$ supergravity theories and harmonic functions," Nucl.Phys. B510 (1998) 247, hep-th/9704147; "General BPS black holes in five dimensions," Mod. Phys. Lett. A13, 239 (1998), hep-th/9708103.

[39] work in progress.

[40] R. Kallosh, A. Linde, M. Shmakova, "Supersymmetric Multiple Basin Attractors", JHEP 9911 (1999) 010

[41] R. Kallosh, "Multivalued Entropy of Supersymmetric Black Holes," JHEP 0001 (2000) 001

[42] S. Ferrara, G. W. Gibbons, R. Kallosh, "Black Holes and Critical Points in Moduli Space," Nucl.Phys. B500 (1997) 75, hep-th/9702103

[43] A. Chamseddine, S. Ferrara, G. Gibbons, R. Kallosh, Phys.Rev. D55 (1997) 3647, hep-th/9610155.

[44] K. Behrndt, A. H. Chamseddine and W. A. Sabra, "BPS black holes in N=2 five dimensional ADS supergravity," Phys. Lett. B422,(1998) 97, hep-th/9807187.

[45] R. Britto-Pacumio, J. Michelson, A. Strominger and A. Volovich, "Lectures on superconformal quantum mechanics and multi-black hole moduli spaces", hep-th/9911066.

[46] S. Ferrara, G. Gibbons and R. Kallosh, "Black holes and critical points in moduli space", hep-th/9702103.

[47] F. Denef, "Supergravity flows and D-brane stability", hep-th/0005049

[48] S. Wu, "On the Instanton Complex of Holomorphic Morse Theory," math.AG/9806118.

[49] M. Gunaydin, M. Zagermann, "The Gauging of Five-dimensional, N=2 MaxwellEinstein Supergravity Theories coupled to Tensor Multiplets," Nucl.Phys. B572 (2000) 131.

[50] B. R. Greene, K. Schalm and G. Shiu, "Dynamical topology change in M theory," hep-th/0010207.

[51] J. Bagger and E. Witten, Nucl.Phys. B222 (1983) 1.

[52] Y.-K. E. Cheung, O.J. Ganor, M. Krogh, "On the Twisted $(2,0)$ and Little String Theories," Nucl. Phys. B536 (1998) 175, hep-th/9805045.

[53] E. Bergshoeff, R. Kallosh, A. Van Proeyen, "Supersymmetry in singular spaces," JHEP 0010 (2000) 033, hep-th/0007044. 